\title{
Dual-mode Explicit Output-feedback Predictive Control Based on Neural Network Models ${ }^{\star}$
}

\author{
Alexandra Grancharova* Juš Kocijan ${ }^{* *, * * *}$ \\ Tor A. Johansen ${ }^{* * * *}$ \\ ${ }^{*}$ Institute of Control and System Research, Bulgarian Academy of \\ Sciences, Acad. G. Bonchev str., Bl.2, P.O.Box 79, Sofia 1113, \\ Bulgaria (e-mail: alexandra.grancharova@abv.bg) \\ ** Department of Systems and Control, Jozef Stefan Institute, \\ Jamova 39, 1000 Ljubljana,Slovenia (e-mail: jus.kocijan@ijs.si) \\ *** Centre for systems and information technologies, University of \\ Nova Gorica, Vipavska 13, 5000 Nova Gorica, Slovenia \\ **** Department of Engineering Cybernetics, Norwegian University of \\ Science and Technology, 7491 Trondheim, Norway \\ (e-mail: Tor.Arne.Johansen@itk.ntnu.no)
}

\begin{abstract}
This paper applies an approximate multi-parametric Nonlinear Programming approach to explicitly solve output-feedback Nonlinear Model Predictive Control (NMPC) problems for constrained nonlinear systems described by black-box models. In particular, neural network models are used and the optimal regulation problem is considered. A dual-mode control strategy is employed in order to achieve an offset-free closed-loop response in the presence of bounded disturbances and/or model errors. The approach is applied to design an explicit NMPC for regulation of a $\mathrm{pH}$ maintaining system.
\end{abstract}

Keywords: Predictive control, Dual-mode control, Neural network models, Piecewise linear controllers.

\section{INTRODUCTION}

Nonlinear Model Predictive Control (NMPC) involves the solution at each sampling instant of a finite horizon optimal control problem subject to nonlinear system dynamics and state and input constraints (Chen and Allgöwer (1998)). There exists a number of NMPC approaches based on various black-box models e.g. based on neural network models (e.g. Nørgaard et al. (2000)), fuzzy models (e.g. Espinosa et al. (2005)), local model networks (e.g. Garcia et al. (2008)), Gaussian Process models (e.g. Kocijan and Murray-Smith (2005)). The common feature of these NMPC approaches is that an on-line optimization needs to be performed in order to compute the optimal control input. Consequently, the computation is time consuming and the real-time NMPC implementation is limited to processes where the sampling time is sufficient to support the computational needs. However, the on-line computational complexity can be circumvented with an explicit approach to NMPC, where the only computation performed on-line would be a simple function evaluation. The benefits of an explicit solution, in addition to the efficient on-line computations, include also verifiability of the implementation. Several approaches to explicit solution of NMPC problems

\footnotetext{
* This work was financed by the National Science Fund of the Ministry of Education, Youth and Science of Republic of Bulgaria, contract DO02-94/14.12.2008 and the Slovenian Research Agency, contract BI-BG/09-10-005 ("Application of Gaussian processes to the modeling and control of complex stochastic systems").
}

have been suggested. In Johansen (2002), Johansen (2004), Grancharova et al. (2007), approaches for off-line computation of explicit sub-optimal piecewise linear (PWL) predictive controllers for nonlinear systems with state and input constraints have been developed, based on the multiparametric Nonlinear Programming (mp-NLP) ideas (Fiacco (1983)). It has been shown that for convex mp-NLP problems, it is straightforward to impose tolerances on the level of approximation such that theoretical properties like asymptotic stability of the sub-optimal feedback controller can be ensured (Johansen (2004), Bemporad and Filippi (2006)). In Grancharova et al. (2007), practical computational methods to handle non-convex mp-NLP problems have been suggested. Algorithms for solving mp-NLP problems, including the non-convex case, are described also in Pistikopoulos et al. (2007). The mentioned methods for explicit NMPC are based on first-principles models and they assume that the state variables can be measured. In addition to these methods, there exists another group of approaches for off-line computation of sub-optimal controllers, where the optimal solution is approximated by means of neural networks (Parisini and Zoppoli (1995), Bertsekas and Tsitsiklis (1998), Åkesson and Toivonen (2006)).

This paper suggests an approximate mp-NLP approach to explicit solution of NMPC problems for constrained nonlinear systems described by neural network NARX models (Chen et al. (1990)). The approach builds an orthogonal 
search tree structure of the regressor space partition and consists in constructing a PWL approximation to the optimal control sequence. A dual-mode control strategy is proposed in order to achieve an offset-free closed-loop response in the presence of bounded disturbances and/or model errors near the system equilibrium. It is similar to the dual-mode control concept in Michalska and Mayne (1993), however here black-box models are considered and an explicit solution of the NMPC problem is sought. The main motivations behind the dual-mode control strategy are the following. First, it may be beneficial to use a separate linear model in a neighborhood of the equilibrium, since the nonlinear black-box model may not have accurate linearizations, and the requirement for accurate control is highest at the equilibrium. Second, it leads to a reduced complexity of the explicit NMPC compared to augmenting the nonlinear model with an integrator to achieve an integral action directly in the NMPC.

\section{FORMULATION OF THE NN-NMPC PROBLEM}

\subsection{Modelling with neural network NARX models}

Consider a nonlinear dynamical system with input $u \in \mathbb{R}^{m}$ and output $y \in \mathbb{R}^{p}$ and let $U=[u(1), u(2), \ldots, u(M)]$ and $Y=[y(1), y(2), \ldots, y(M)]$ be sets of observed values of $u$ and $y$ to the number of $M$. Based on these data, the dynamics of the system can be described with a neural network NARX model (Chen et al. (1990)), where the future predicted output $y(i+1)$ depends on previous estimated outputs, as well as on previous control inputs:

$$
\begin{aligned}
& y(i+1)=f(z(i), \theta) \\
& z(i)=[y(i), y(i-1), \ldots, y(i-L), \\
& u(i), u(i-1), \ldots, u(i-L)]
\end{aligned}
$$

Here, $L$ is a given lag, $i$ is the consecutive index of data samples $(i \geq L), z(i)$ is the regressor vector, $f$ is the function realized by the neural network model, and $\theta$ is a vector of parameters. The function $f$ is a concatenation of two mappings: one that takes the past values of the observed inputs and outputs and maps them into the regressor vector and one that takes this vector to the space of the outputs. The nonlinear mapping from the regressor space to the output space can be of various kinds. We will use neural network with sigmoid basis functions in the hidden layer and linear basis functions in the output layer. It is called Multilayer Perceptron (MLP) (Nørgaard et al. (2000)) and can be used as an universal approximator. The parameters of the MLP are the weights of its units. After the structure (number of layers and units) is determined, the model parameters are obtained with optimization, based on a chosen cost function (usually a least squares combination of errors between estimated and measured output signals $E=\frac{1}{2 M} \sum_{i=1}^{M}\|y(i)-\hat{y}(i \mid \theta)\|^{2}$, where $\hat{y}(i \mid \theta)$ is estimated output signal).

\subsection{Formulation of the NN-NMPC problem}

Consider the discrete-time nonlinear system:

$$
\begin{aligned}
& x(t+1)=h(x(t), u(t)) \\
& y(t)=g(x(t), u(t))
\end{aligned}
$$

where $x(t) \in \mathbb{R}^{n}, u(t) \in \mathbb{R}^{m}$, and $y(t) \in \mathbb{R}^{p}$ are the state, input and output vectors, and $h: \mathbb{R}^{n} \times \mathbb{R}^{m} \rightarrow \mathbb{R}^{n}$ and $g: \mathbb{R}^{n} \times \mathbb{R}^{m} \rightarrow \mathbb{R}^{p}$ are nonlinear functions. Input and output constraints are imposed on the system (3)-(4):

$$
u_{\min } \leq u(t) \leq u_{\max }, y_{\min } \leq y(t) \leq y_{\max }
$$

Assume that the dynamics of the nonlinear system (3)-(4) is approximated with an MLP neural network with NARX structure of the form (1)-(2). Then for $t \geq L$, define a modified regressor vector:

$$
\tilde{z}(t)=\left\{\begin{array}{c}
{[y(t), y(t-1), \ldots, y(t-L),} \\
u(t-1), \ldots, u(t-L)], \text { if } L>0 \\
y(t), \text { if } L=0
\end{array}\right.
$$

where $u(t-1), \ldots, u(t-L)$ and $y(t), y(t-1), \ldots, y(t-L)$ are the measured values of the input $u$ and the output $y$. Thus, $\tilde{z}(t) \in \mathbb{R}^{q}$ with $q=(L+1) p+L m$. Then, the NARX model, used to obtain one-step ahead prediction of the output for $t \geq L$, is represented:

$$
\hat{y}(t+1 \mid \theta)=f_{N N}(\tilde{z}(t), u(t), \theta)
$$

where $f_{N N}$ is the function realized by the neural network (NN) and $\theta$ contains the network weights. Suppose the initial regressor vector $\tilde{z}(t)=\tilde{z}_{t \mid t}$ is known and the control inputs $u(t+k)=u_{t+k}, k=0,1, \ldots, N-1$ are given. Then, the model (7) can be used to obtain the predicted output $y_{t+k+1 \mid t}, k=0,1, \ldots, N-1$ through iterative onestep ahead predictions, where at each step the predicted output value is fed back to the regressor vector:

$$
\begin{aligned}
& y_{t+k+1 \mid t}=f_{N N}\left(\tilde{z}_{t+k \mid t}, u_{t+k}, \theta\right) \\
& \tilde{z}_{t+k \mid t}=\left\{\begin{array}{c}
{\left[y_{t+k \mid t}, y_{t+k-1 \mid t}, \ldots, y_{t+k-L \mid t},\right.} \\
\left.u_{t+k-1}, \ldots, u_{t+k-L}\right], \text { if } L>0 \\
y_{t+k \mid t}, \text { if } L=0
\end{array}\right.
\end{aligned}
$$

It is assumed that there exists $u_{s t}^{\mathrm{NN}} \in \mathbb{R}^{m}$ satisfying $u_{\min } \leq$ $u_{s t}^{\mathrm{NN}} \leq u_{\max }$, and such that $f_{N N}\left(\tilde{z}_{0}, u_{s t}^{\mathrm{NN}}, \theta\right)=0$, where $\tilde{z}_{0}$ is obtained from (9) with $y_{t+k \mid t}=y_{t+k-1 \mid t}=\ldots=$ $y_{t+k-L \mid t}=0, u_{t+k-1}=\ldots=u_{t+k-L}=u_{s t}^{\mathrm{NN}}$. It means that the point $y=0, u=u_{s t}^{\mathrm{NN}}$, is an equilibrium point for the NARX model (7). It is also supposed that $y_{\text {min }}<0<$ $y_{\max }$, which means that this equilibrium point is feasible for (5). Further, let $\tilde{z}^{c}=\tilde{z}-\left[0_{(L+1) p}^{T} u_{s t}^{N N^{T}} \ldots u_{s t}^{N N^{T}}\right]^{T}$, where $0_{(L+1) p}$ is a zero vector with dimension $(L+1) p$.

Consider the optimal regulation problem where the goal is to steer the output $y$ to the origin by minimizing certain performance criterion. Suppose that a full measurement of the regressor vector $\tilde{z}(t)$ is available at the current time $t \geq L$. Then, for the current $\tilde{z}(t)$, the regulation NN$\mathrm{NMPC}$ solves the following optimization problem:

$V^{*}(\tilde{z}(t))=\min _{U} J(U, \tilde{z}(t))$

subject to $\tilde{z}_{t \mid t}=\tilde{z}(t)$ and:

$y_{\min } \leq y_{t+k \mid t} \leq y_{\max }, k=1, \ldots, N$

$u_{\min } \leq u_{t+k} \leq u_{\max }, k=0,1, \ldots, N-1$

$\tilde{z}_{t+N \mid t}^{c} \in \Omega$

$y_{t+k+1 \mid t}=f_{N N}\left(\tilde{z}_{t+k \mid t}, u_{t+k}, \theta\right), k=0,1, \ldots, N-1(14)$ 


$$
\begin{aligned}
\tilde{z}_{t+k \mid t}=\left\{\begin{array}{c}
{\left[y_{t+k \mid t}, y_{t+k-1 \mid t}, \ldots, y_{t+k-L \mid t}\right.} \\
\left.u_{t+k-1}, \ldots, u_{t+k-L}\right], \text { if } L>0 \\
y_{t+k \mid t}, \text { if } L=0
\end{array}\right. \\
k=0,1, \ldots, N-1
\end{aligned}
$$

with $U=\left[u_{t}, u_{t+1}, \ldots, u_{t+N-1}\right]$ and the cost function:

$$
\begin{gathered}
J(U, \tilde{z}(t))=\sum_{k=0}^{N-1}\left[\left\|y_{t+k \mid t}\right\|_{Q}^{2}+\left\|u_{t+k}-u_{s t}^{\mathrm{NN}}\right\|_{R}^{2}\right]+ \\
\left\|y_{t+N \mid t}\right\|_{F}^{2}
\end{gathered}
$$

Here, $N$ is a finite horizon and $Q, R, F \succ 0$. In (13), $\Omega$ is the terminal set defined by $\Omega=\left\{\tilde{z}^{c} \in \mathbb{R}^{q} \mid\left\|\tilde{z}^{c}\right\|^{2} \leq \delta\right\}$ with $\delta>0$. From a stability point of view it is desirable to choose $\delta$ as small as possible (Mayne et al. (2000)). Let $\tilde{z}$ be the value of the regressor vector at the current time $t$. Then, the optimization problem (10)-(16) can be formulated in a compact form as follows:

$$
V^{*}(\tilde{z})=\min _{U} J(U, \tilde{z}) \text { subject to } G(U, \tilde{z}) \leq 0
$$

The problem (17) defines an mp-NLP, since it is NLP in $U$ parameterized by $\tilde{z}$. We remark that the constraints function $G(U, \tilde{z})$ in (17) is implicitly defined by (11)-(15). An optimal solution to this problem is denoted $U^{*}=$ $\left[u_{t}^{*}, u_{t+1}^{*}, \ldots, u_{t+N-1}^{*}\right]$ and the control input is chosen according to the receding horizon policy $u(t)=u_{t}^{*}$. Define the set of $N$-step feasible initial regressor vectors:

$$
Z_{f}=\left\{\tilde{z} \in \mathbb{R}^{q} \mid G(U, \tilde{z}) \leq 0 \text { for some } U \in \mathbb{R}^{N m}\right\}
$$

In parametric programming problems one seeks the solution $U^{*}(\tilde{z})$ as an explicit function of the parameters $\tilde{z}$ in some set $\underline{Z} \subseteq Z_{f} \subseteq \mathbb{R}^{q}($ Fiacco $(1983))$.

\section{APPROXIMATE MP-NLP APPROACH}

In Grancharova et al. (2007), a computational method for constructing an explicit piecewise linear (PWL) approximate solution of state-space NMPC problems has been suggested. Here, the approximate mp-NLP approach is applied to explicitly solve the output-feedback NN-NMPC problem formulated in the previous section and it is given only in brief. Let $Z \subset \mathbb{R}^{q}$ be a hyper-rectangle where we seek to approximate the optimal solution $U^{*}(\tilde{z})$ to problem (17). It is required that the regressor space partition is orthogonal and can be represented as a $k-d$ tree. The main idea of the approximate mp-NLP approach is to construct a PWL approximation $\widehat{U}(\tilde{z})$ to $U^{*}(\tilde{z})$ on $Z$, where the constituent affine functions are defined on hyper-rectangles covering $Z$. The computation of an affine regressor feedback associated to a given region $Z_{0}$ includes the following steps. First, a close-to-global solution of problem (17) is computed at a set of points $V_{0}=\left\{v_{0}, v_{1}, v_{2}, \ldots\right.$, $\left.v_{N_{1}}\right\} \subset Z_{0}$. Then, based on the solutions at these points, a local linear approximation $\widehat{U}_{0}(\tilde{z})=K_{0} \tilde{z}+g_{0}$ to the close-to-global solution $U^{*}(\tilde{z})$, valid in the whole hyperrectangle $Z_{0}$, is determined by applying the procedure in Grancharova et al. (2007):

Procedure 1. Consider any hyper-rectangle $Z_{0} \subseteq Z$ with a set of points $V_{0}=\left\{v_{0}, v_{1}, v_{2}, \ldots, v_{N_{1}}\right\} \subset Z_{0}$. Compute $K_{0}$ and $g_{0}$ by solving the following NLP:

$$
\begin{gathered}
\min _{K_{0}, g_{0}} \sum_{i=0}^{N_{1}}\left(J\left(K_{0} v_{i}+g_{0}, v_{i}\right)-V^{*}\left(v_{i}\right)+\right. \\
\left.\alpha\left\|K_{0} v_{i}+g_{0}-U^{*}\left(v_{i}\right)\right\|^{2}\right) \\
\text { subject to } G\left(K_{0} v_{i}+g_{0}, v_{i}\right) \leq 0, \forall v_{i} \in V_{0}
\end{gathered}
$$

In (19), $J\left(K_{0} v_{i}+g_{0}, v_{i}\right)$ is the sub-optimal cost, $V^{*}\left(v_{i}\right)$ denotes the cost corresponding to the close-to-global solution $U^{*}\left(v_{i}\right)$, i.e. $V^{*}\left(v_{i}\right)=J\left(U^{*}\left(v_{i}\right), v_{i}\right)$, and the parameter $\alpha$ is a weighting coefficient (tuned in an ad-hoc fashion). Note that the computed linear regressor feedback $\widehat{U}_{0}(\tilde{z})=$ $K_{0} \tilde{z}+g_{0}$ satisfies the constraints in problem (17) only at the discrete set of points $V_{0} \subset Z_{0}$. After the feedback $\widehat{U}_{0}(\tilde{z})$ has been determined, an estimate $\widehat{\varepsilon}_{0}$ of the maximal cost function approximation error in $Z_{0}$ is computed as follows:

$$
\widehat{\varepsilon}_{0}=\max _{i \in\left\{0,1,2, \ldots, N_{1}\right\}}\left(J\left(K_{0} v_{i}+g_{0}, v_{i}\right)-V^{*}\left(v_{i}\right)\right)
$$

If $\widehat{\varepsilon}_{0}>\bar{\varepsilon}$, where $\bar{\varepsilon}>0$ is the specified tolerance of the approximation error, the region $Z_{0}$ is divided and the procedure is repeated for the new regions. The detailed approximate mp-NLP algorithm is given in Grancharova et al. (2007) (where the state space should be replaced by the regressor space). The algorithm terminates with a PWL function $\widehat{U}(\tilde{z})=\left[\widehat{u}_{0}(\tilde{z}), \widehat{u}_{1}(\tilde{z}), \ldots, \widehat{u}_{N-1}(\tilde{z})\right]$ that is defined on an inner approximation $Z_{\Pi}$ of the set $Z \cap Z_{f}$.

\section{EXPLICIT DUAL-MODE CONTROLLER}

Generally, it will be difficult to guarantee that the local linearization at a nominal equilibrium point of an $\mathrm{NN}$ ARX model is accurate. The inaccuracies of the model may result in a steady-state offset of the explicit NNNMPC controller. Here, a dual-mode control strategy is proposed which aims at achieving an offset-free closed-loop response in the presence of bounded disturbances and/or model errors. With this strategy, the control is performed by the explicit NN-NMPC controller when the system is far from equilibrium, and by a Linear Quadratic Regulator (LQR) with integral action when it is close to equilibrium.

\subsection{Design of LQR with integral action in a neighborhood of the equilibrium}

Consider a linear ARX model (Ljung and Glad (1994)):

$$
\begin{aligned}
y(t+1)= & A_{1} y(t)+A_{2} y(t-1)+\ldots+A_{l+1} y(t-l)+ \\
& B_{1}\left(u(t)-u_{s t}^{*}\right)+B_{2}\left(u(t-1)-u_{s t}^{*}\right)+\ldots \\
& +B_{l+1}\left(u(t-l)-u_{s t}^{*}\right)
\end{aligned}
$$

that is valid in a neighborhood of the equilibrium $y=0$, $u=u_{s t}^{*}$ of the considered nonlinear dynamical system (3)(4). In (22), the matrices $A_{i} \in \mathbb{R}^{p \times p}$ and $B_{i} \in \mathbb{R}^{p \times m}$, $i=1,2, \ldots, l+1$ contain the coefficients of the model, and $l$ is a given lag. To estimate the parameters of the model (22), the least squares estimation method or the four-stage instrumental variable method can be applied (Ljung and Glad (1994)). Based on the linear ARX model, an LQR that will regulate the system (22) to the origin, is designed. In order to achieve an offset-free performance, the model (22) is augmented with the following output $y_{\text {int }} \in \mathbb{R}^{p}$, which takes into account the integral error: 


$$
y_{\text {int }}(t+1)=y_{\text {int }}(t)+T_{s} y(t)
$$

where $T_{s}$ is the sampling time. Let $u_{e}(t) \equiv u(t)-u_{s t}^{*}$. Then, the extended system with input $u_{e}$ and output $y_{e}=\left[y, y_{i n t}\right]$ is described by the linear ARX model:

$$
\begin{gathered}
y_{e}(t+1)=A_{1}^{e} y_{e}(t)+A_{2}^{e} y_{e}(t-1)+\ldots+A_{l+1}^{e} y_{e}(t-l)+ \\
B_{1}^{e} u_{e}(t)+B_{2}^{e} u_{e}(t-1)+\ldots+B_{l+1}^{e} u_{e}(t-l),
\end{gathered}
$$

where $A_{1}^{e}=\left[\begin{array}{cc}A_{1} & 0_{p} \\ T_{s} I_{p} & I_{p}\end{array}\right], A_{i}^{e}=\left[\begin{array}{cc}A_{i} & 0_{p} \\ 0_{p} & 0_{p}\end{array}\right], i=2,3, \ldots, l+$ $1, B_{i}^{e}=\left[\begin{array}{c}B_{i} \\ 0_{p, m}\end{array}\right], i=1,2, \ldots, l+1$. Here, $I_{p}$ is the $p$ dimensional identity matrix, $0_{p}$ is the $p$-dimensional square zero matrix, and $0_{p, m}$ is the $p \times m$-dimensional zero matrix. The following regressor vector is introduced:

$$
\tilde{z}_{e}(t)=\left\{\begin{array}{l}
{\left[y_{e}(t), y_{e}(t-1), \ldots, y_{e}(t-l),\right.} \\
\left.u_{e}(t-1), u_{e}(t-2), \ldots, u_{e}(t-l)\right], \text { if } l>0 \\
y_{e}(t), \text { if } l=0
\end{array}\right.
$$

Thus, $\tilde{z}_{e}(t) \in \mathbb{R}^{q_{e}}$ with $q_{e}=(l+1) 2 p+l m$. This vector can be also represented as $\tilde{z}_{e}(t)=\left[z_{1}(t), z_{2}(t), \ldots, z_{l+l+1}(t)\right]$, where $z_{1}(t), \ldots, z_{l+1}(t)$ are the shifted values of $y_{e}$ and $z_{l+2}(t), \ldots, z_{l+l+1}(t)$ are the shifted values of $u_{e}$. The following relations hold:

$$
\begin{aligned}
& y_{e}(t+1)=z_{1}(t+1) \\
& z_{1}(t)= y_{e}(t)=z_{2}(t+1) \\
& z_{2}(t)= y_{e}(t-1)=z_{3}(t+1) \\
& \vdots \\
& z_{l}(t)= y_{e}(t-l+1)=z_{l+1}(t+1) \\
& z_{l+1}(t)= y_{e}(t-l) \\
& z_{l+2}(t)=u_{e}(t)=z_{l+2}(t+1) \\
& z_{l+3}(t)=u_{e}(t-1)=z_{l+3}(t+1) \\
&= u_{e}(t-2)=z_{l+4}(t+1) \\
& z_{l+l}(t)= u_{e}(t-l+1)=z_{l+l+1}(t+1) \\
& z_{l+l+1}(t)= u_{e}(t-l)
\end{aligned}
$$

Then, the system (24) can be represented:

$$
\tilde{z}_{e}(t+1)=\tilde{A}^{e} \tilde{z}_{e}(t)+\tilde{B}^{e} u_{e}(t)
$$

For $l>0$, the matrices $\tilde{A}^{e}$ and $\tilde{B}^{e}$ in (28) are given by:

$$
\tilde{A}^{e}=\left[\begin{array}{ccccccccc}
A_{1}^{e} & A_{2}^{e} & \ldots & A_{l}^{e} & A_{l+1}^{e} & B_{2}^{e} & \ldots & B_{l}^{e} & B_{l+1}^{e} \\
I_{2 p} & 0_{2 p} & \ldots & 0_{2 p} & 0_{2 p} & 0_{2 p, m} & \ldots & 0_{2 p, m} & 0_{2 p, m} \\
0_{2 p} & I_{2 p} & \ldots & 0_{2 p} & 0_{2 p} & 0_{2 p, m} & \ldots & 0_{2 p, m} & 0_{2 p, m} \\
\vdots & & & & & & & & \\
0_{2 p} & 0_{2 p} & \ldots & I_{2 p} & 0_{2 p} & 0_{2 p, m} & \ldots & 0_{2 p, m} & 0_{2 p, m} \\
0_{m, 2 p} & 0_{m, 2 p} & \ldots & 0_{m, 2 p} & 0_{m, 2 p} & 0_{m} & \ldots & 0_{m} & 0_{m} \\
0_{m, 2 p} & 0_{m, 2 p} & \ldots & 0_{m, 2 p} & 0_{m, 2 p} & I_{m} & \ldots & 0_{m} & 0_{m} \\
\vdots & & & & & & & & \\
0_{m, 2 p} & 0_{m, 2 p} & \ldots & 0_{m, 2 p} & 0_{m, 2 p} & 0_{m} & \ldots & I_{m} & 0_{m}
\end{array}\right]
$$

$$
\tilde{B}^{e}=\left[\begin{array}{lllllllll}
B_{1}^{e} & 0_{2 p, m} & 0_{2 p, m} & \ldots & 0_{2 p, m} & I_{m} & 0_{m} & \ldots & 0_{m}
\end{array}\right]^{T}
$$

In (29), (30), $I_{2 p}$ and $I_{m}$ are identity matrices, $0_{2 p}$ and $0_{m}$ are square zero matrices, and $0_{2 p, m}$ and $0_{m, 2 p}$ are zero matrices with dimensions $2 p \times m$ and $m \times 2 p$ respectively. If $l=0$, then $\tilde{A}^{e}=A_{1}^{e}$ and $\tilde{B}^{e}=B_{1}^{e}$.

The unconstrained LQR problem for system (28) solves the following optimization problem:

$$
\min _{\left\{u_{e}(t), u_{e}(t+1), \ldots\right\}} \sum_{k=0}^{\infty}\left[\left\|\tilde{z}_{e}(t+k)\right\|_{Q_{e}}^{2}+\left\|u_{e}(t+k)\right\|_{R_{e}}^{2}\right](31)
$$

where $Q_{e}, R_{e} \succ 0$. The solution to (31) is the linear feedback control law:

$$
u_{e}(t+k)=-K \tilde{z}_{e}(t+k), k \geq 0,
$$

where the controller gain $K$ is given by Ogata (1995):

$$
\begin{aligned}
K= & \left(\tilde{B}^{e T} P \tilde{B}^{e}+R_{e}\right)^{-1} \tilde{B}^{e T} P \tilde{A}^{e} \\
P= & \tilde{A}^{e T} P \tilde{A}^{e}+Q_{e}- \\
& \tilde{A}^{e T} P \tilde{B}^{e}\left(\tilde{B}^{e T} P \tilde{B}^{e}+R_{e}\right)^{-1}\left(\tilde{A}^{e T} P \tilde{B}^{e}\right)^{T}
\end{aligned}
$$

By taking into account that $u_{e}(t) \equiv u(t)-u_{s t}^{*}$, it follows from (32) that the control input applied to the system is:

$$
u(t+k)=-K \tilde{z}_{e}(t+k)+u_{s t}^{*}, k \geq 0
$$

\subsection{Explicit dual-mode controller}

Consider the closed-loop system:

$$
\tilde{z}_{e}(t+k)=\left(\tilde{A}^{e}-\tilde{B}^{e} K\right) \tilde{z}_{e}(t+k-1), k \geq 0,
$$

where $\tilde{z}_{e}(t+k)$ is defined by (25) if $t$ is replaced by $t+k$. Assume that $A_{c l}=\tilde{A}^{e}-\tilde{B}^{e} K$ is strictly Hurwitz. Let $\Gamma_{e}=\left\{\tilde{z}_{e} \in \mathbb{R}^{q_{e}} \mid \tilde{z}_{e}^{T} S \tilde{z}_{e} \leq \sigma\right\}$ with $S \succ 0, \sigma>0$, be a positively invariant admissible set for the system (36). It means that $\forall \tilde{z}_{e}(t) \in \Gamma_{e}, \tilde{z}_{e}(t+k) \in \Gamma_{e}, \forall k>0$ and:

$$
\begin{aligned}
& y_{\min }<\left[\Psi 0_{2 p} \ldots 0_{2 p} 0_{m} \ldots 0_{m}\right] \tilde{z}_{e}(t+k)<y_{\max }, k \geq 0 \\
& u_{\min }<-K \tilde{z}_{e}(t+k)+u_{s t}^{*}<u_{\max }, k \geq 0
\end{aligned}
$$

where $\Psi=\left[I_{p} 0_{p}\right]$ and $I_{p}, 0_{p}, 0_{2 p}, 0_{m}$ are defined above. $\Gamma_{e}$ can be determined in a way similar to Lemma 1 in Chen and Allgöwer (1998). If $S$ satisfies the Lyapunov equation:

$$
A_{c l}^{T} S A_{c l}-S=-\mu S-Q_{e}-K^{T} R_{e} K
$$

for some $\mu>0$, then there exists a constant $\sigma>0$ such that the set $\Gamma_{e}$ is a positively invariant admissible set for the system (36). For $l \leq L$, let $\widetilde{\Gamma}_{e}^{1}=\left\{\xi \in \mathbb{R}^{\tilde{q}_{e}} \mid \xi^{T} \widetilde{S}_{1} \xi \leq\right.$ $\left.\tilde{\sigma}_{1}\right\}$ with $\widetilde{S}_{1} \succ 0, \tilde{\sigma}_{1}>0$ be the orthogonal projection of $\Gamma_{e}$ onto $\mathbb{R}^{\tilde{q}_{e}}, \tilde{q}_{e}=(l+1) p+l m$, by omitting all integrator elements from the regressor vector $\tilde{z}_{e}$. Let $\widetilde{\Omega}^{1}=\{\zeta \in$ $\left.\mathbb{R}^{\tilde{q}_{e}} \mid \zeta^{T} \widetilde{S}_{1} \zeta \leq\left\|\widetilde{S}_{1}\right\| \tilde{\delta}_{1}\right\}$ be the orthogonal projection of the terminal set $\Omega$ onto $\mathbb{R}^{\tilde{q}_{e}}$, where $\left\|\widetilde{S}_{1}\right\|$ is the induced norm of matrix $\widetilde{S}_{1}$. Then, it is required $\left\|\widetilde{S}_{1}\right\| \tilde{\delta}_{1}<\tilde{\sigma}_{1}$, so that $\widetilde{\Omega}^{1} \subset \widetilde{\Gamma}_{e}^{1}$. For $l>L$, let $\widetilde{\Gamma}_{e}^{2}=\left\{\xi \in \mathbb{R}^{q} \mid \xi^{T} \widetilde{S}_{2} \xi \leq \tilde{\sigma}_{2}\right\}$ with $\widetilde{S}_{2} \succ 0, \tilde{\sigma}_{2}>0$ be the orthogonal projection of $\Gamma_{e}$ onto $\mathbb{R}^{q}, q=(L+1) p+L m$, by omitting all integrator elements and the elements $y(t-L-1), \ldots, y(t-l)$ from 
$\tilde{z}_{e}$. Let $\widetilde{\Omega}^{2}=\left\{\zeta \in \mathbb{R}^{q} \mid \zeta^{T} \widetilde{S}_{2} \zeta \leq\left\|\widetilde{S}_{2}\right\| \tilde{\delta}_{2}\right\}$, with $\tilde{\delta}_{2}>0$ be a set such that $\Omega \subseteq \widetilde{\Omega}^{2}$. Similar to above it is required $\left\|\widetilde{S}_{2}\right\| \tilde{\delta}_{2}<\tilde{\sigma}_{2}$, so that $\widetilde{\Omega}^{2} \subset \widetilde{\Gamma}_{e}^{2}$.

In order to define the dual-mode controller, the regressor vector, associated to the system (36), is introduced:

$$
\tilde{z}_{r}(t)=\left\{\begin{array}{l}
{\left[\Psi y_{e}(t), \Psi y_{e}(t-1), \ldots, \Psi y_{e}(t-l),\right.} \\
\left.u_{e}(t-1)+u_{s t}^{*}, \ldots, u_{e}(t-l)+u_{s t}^{*}\right], \text { if } l>0(40) \\
\Psi y_{e}(t), \text { if } l=0
\end{array}\right.
$$

where $\Psi$ is defined above. Thus, $\tilde{z}_{r}(t) \in \mathbb{R}^{q_{r}}$ with $q_{r}=(l+$ 1)p $p+\operatorname{lm}$. Let $\Gamma_{r} \in \mathbb{R}^{q_{r}}$ be the orthogonal projection of $\Gamma_{e}$ onto $\mathbb{R}^{q_{r}}$, specified by (40) (note that $q_{r}<q_{e}$ ). Further, for $l=L$, it is required $\Gamma_{r} \subset Z_{\Pi} \subset \mathbb{R}^{q}$. For $l<L$, $\Gamma_{r} \subset \widetilde{Z}_{\Pi} \subset \mathbb{R}^{q_{r}}$, where $\widetilde{Z}_{\Pi}$ is the orthogonal projection of $Z_{\Pi}$ onto $\mathbb{R}^{q_{r}}$, obtained by omitting the regressors with lag larger than $l$. For $l>L, \widetilde{\Gamma}_{r} \subset Z_{\Pi} \subset \mathbb{R}^{q}$, where $\widetilde{\Gamma}_{r}$ is the orthogonal projection of $\Gamma_{r}$ onto $\mathbb{R}^{q}$, obtained by omitting the regressors with lag larger than $L$.

Let $\tilde{z}, \tilde{z}_{e}$, and $\tilde{z}_{r}$ be the values of the regressor vectors (6), (25), and (40) at the current time $t$. Then, the explicit dual-mode controller is defined as follows:

$$
u_{d} \triangleq\left\{\begin{array}{l}
\widehat{u}_{0}(\tilde{z}), \text { if } \tilde{z}_{r} \notin \Gamma_{r} \\
-K \tilde{z}_{e}+u_{s t}^{*}, \text { if } \tilde{z}_{r} \in \Gamma_{r}
\end{array}\right.
$$

The expression in the first row of (41) means that the control is performed by the explicit NN-NMPC controller when the system is far from equilibrium. The expression in the second row implies that the control will be switched to the LQR when $\tilde{z}_{r}$ enters the set $\Gamma_{r}$ and the LQR will continue controlling the system until $\tilde{z}_{r}$ leaves this set due to a large disturbance, for example. The integrator output $y_{\text {int }}$ is used only when $\tilde{z}_{r} \in \Gamma_{r}$. In the case when $\tilde{z}_{r} \notin \Gamma_{r}$, $y_{\text {int }}$ is set to zero and not used.

If the NN ARX model describes exactly the system dynamics far from the origin (outside the set $\Gamma_{r}$ ) and the problem (17) is convex, then the closed-loop system stability can be ensured by conditions similar to those in Johansen (2004). In presence of model errors far from the origin, it would be necessary to apply approaches to explicit robust NMPC (Grancharova and Johansen (2009)). If the problem (17) is non-convex, then the closed-loop stability can not be guaranteed, but it can be verified by off-line simulations.

\section{SIMULATION EXAMPLE}

Here, an explicit dual-mode controller for regulation of a $\mathrm{pH}$ maintaining system is designed. The system (described in details in Henson and Seborg (1994)) consists of an acid stream $\left(Q_{1}\right)$, buffer stream $\left(Q_{2}\right)$ and base stream $\left(Q_{3}\right)$ that are mixed in a tank $\left(\mathrm{T}_{1}\right)$. Prior to mixing, the acid stream enters another tank $\left(\mathrm{T}_{2}\right)$. The acid and buffer flow rates are assumed to be constant. The effluent $\mathrm{pH}$ is the measured variable, which is controlled by manipulating the base flow rate. In Henson and Seborg (1994), a dynamic model of the system is derived, which has the state, input and output variables:

$$
x=\left[\begin{array}{lll}
W_{a 4} & W_{b 4} & h_{1}
\end{array}\right]^{T}, \tilde{u}=Q_{3}, \tilde{y}=\mathrm{pH},
$$

where $W_{a 4}$ and $W_{b 4}$ are the effluent reaction invariants, and $h_{1}$ is the liquid level in tank $\mathrm{T}_{1}$. The obtained state space model has the form:

$$
\dot{x}=\tilde{f}(x)+\tilde{g}(x) \tilde{u}, c(x, \tilde{y})=0
$$

\subsection{ARX model identification}

Neural network ARX model identification The identification and the validation of the $\mathrm{NN}$ model of the $\mathrm{pH}$ maintaining system is based on simulation data, generated with the model (43), where the liquid level $h_{1}$ in tank $\mathrm{T}_{1}$ is assumed to be constant. Thus, it is presumed that a controller has been already designed to keep the level $h_{1}$ on the nominal value $h_{1}^{*}=14[\mathrm{~cm}]$ by manipulating the exit flow rate $Q_{4}$. Based on the responses obtained with the model (43), a sampling time of 25 [s] was selected. The identification signal was generated from a uniform random distribution and a rate of change of the signal of $50[\mathrm{~s}]$. The validation signal was obtained using a generator of random noise with uniform distribution and a rate of change of the signal of $500[\mathrm{~s}]$. The NN ARX model has the form:

$$
\begin{aligned}
& y(t+1)=f_{N N}(\tilde{z}(t), u(t), \theta) \\
& \tilde{z}(t)=[y(t), y(t-1), y(t-2), u(t-1), u(t-2)]
\end{aligned}
$$

It should be noted that in difference to the model (43) where $\tilde{y}=\mathrm{pH}$, in the $\mathrm{NN}$ model (44)-(45) the output variable $y$ represents the deviation of the $\mathrm{pH}$ from the desired set point $\mathrm{pH}_{s p}=4.8$, i.e. $y=\mathrm{pH}-\mathrm{pH}_{s p}$. The data used for identification of the NN model (44)-(45) and for validation of its performance were scaled to zero mean and variance 1 . This means that the control input $u(t)$ and the output $y(t)$ in the NN model can take both positive and negative values. The hidden layer of the NN model has thirteen neurons with sigmoid activation functions and the output neuron has a linear activation function.

Linear ARX model identification The equilibrium point of the $\mathrm{pH}$ maintaining system (43) is $\tilde{y}=4.8, \tilde{u}_{s t}^{*}=$ $10.94[\mathrm{ml} / \mathrm{s}]$ (respectively $y=0, u_{s t}^{*}=0.1732$ after scaling). A validation of the obtained NN ARX model near this point showed that it is not accurate. In order to obtain accurate predictions when the output variable is close to zero, the following linear ARX model is identified:

$$
y(t+1)=0.7704 y(t)+0.0539\left(u(t)-u_{s t}^{*}\right)
$$

\subsection{Design of explicit dual-mode controller}

The approach described in sections 3 and 4 is applied to design an explicit dual-mode controller for the $\mathrm{pH}$ maintaining system based on its NN model (44)-(45) and linear ARX model (46). First, the mp-NLP algorithm in Grancharova et al. (2007) is applied to design an explicit approximate NN-NMPC controller. The control input constraint imposed on the system is $-0.4 \leq u \leq 0.4$. The horizon is $N=8$ and the terminal constraint in the NN-NMPC problem is $\tilde{z}_{t+N \mid t}^{c} \in \Omega$, where $\Omega=\left\{\tilde{z}^{c} \in\right.$ $\left.\mathbb{R}^{5} \mid\left\|\tilde{z}^{c}\right\|^{2} \leq 0.05\right\}$. The weighting matrices in the cost function (16) are $Q=10, R=1, F=10$. The regressor space to be partitioned is defined by $Z=([-1.2 ; 1.2] \times$ $[-1.2 ; 1.2] \times[-1.2 ; 1.2] \times[-0.4 ; 0.4] \times[-0.4 ; 0.4])$. The cost function approximation tolerance is chosen as $\bar{\varepsilon}\left(Z_{0}\right)=$ $\max \left(\bar{\varepsilon}_{a}, \bar{\varepsilon}_{r} \min _{\tilde{z} \in Z_{0}} V^{*}(\tilde{z})\right)$, where $\bar{\varepsilon}_{a}=0.005$ and $\bar{\varepsilon}_{r}=0.1$. The partition has 5512 regions and 33 arithmetic operations are needed in real-time to compute the control input. 
Further, an unconstrained LQR is designed, which is used in a neighborhood of the origin:

$$
u_{e}=-K \tilde{z}_{e}=-k_{1} y-k_{2} y_{\text {int }}, K=[0.7994,0.0069](47)
$$

This control law solves the optimization problem (31) with weighting matrices $Q_{e}=\operatorname{diag}\{10,0.0005\}, R_{e}=10$. Then, the explicit dual-mode controller for the $\mathrm{pH}$ maintaining system is defined according to (41) with $\Gamma_{r}=\left\{\tilde{z}_{r} \in\right.$ $\left.\mathbb{R} \mid \tilde{z}_{r}^{2} \leq 0.09\right\}$, where $\tilde{z}_{r}(t)=y(t)$.

In order to study the robustness of the explicit dual-mode controller against model inaccuracies, its performance is simulated in closed-loop with the first-principles model (43). It is assumed that there are persistent disturbances in the acid and the buffer flow rates. In addition to the explicit dual-mode controller, a second controller (an LQR) is applied, which keeps the liquid level $h_{1}$ on the nominal value $h_{1}^{*}=14[\mathrm{~cm}]$ by manipulating the exit flow rate $Q_{4}$. The obtained trajectories of the control input $u$ and the output variable $y$ are shown in Fig. 1 . It can be seen from Fig. 1 that the output variable is steered to the origin despite of the presence of persistent disturbances and the control input achieves a new equilibrium value $u_{s t}^{*}=0.2380$. The depicted response has a typical amount of performance degradation being representative for other initial conditions and scenarios.

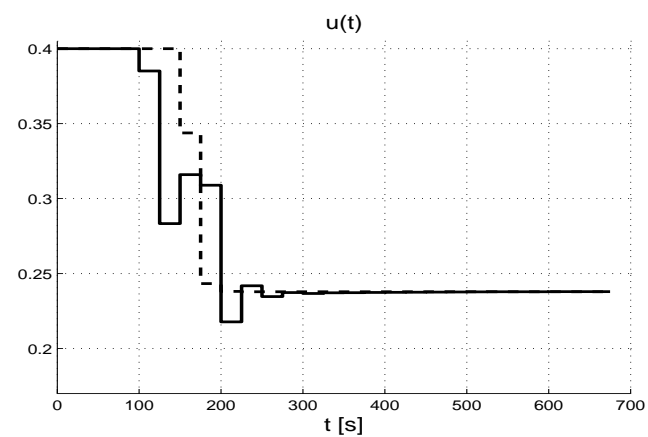

$y(t)$

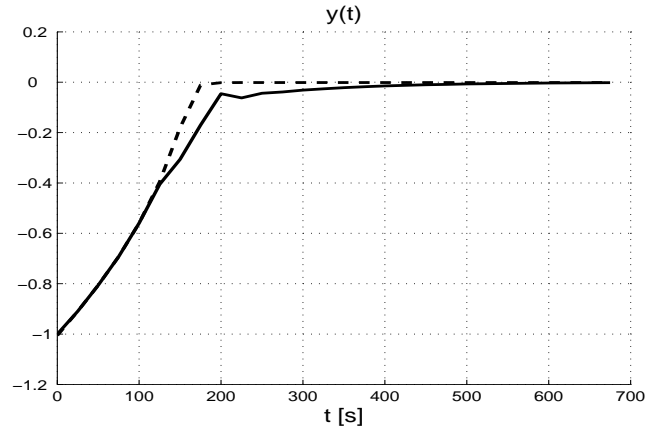

Fig. 1. Control input $u$ and output variable $y$ obtained with the explicit dual-mode controller. The solid curves are with the approximate explicit NN-NMPC and the dashed curves are with the exact NN-NMPC.

\section{REFERENCES}

Åkesson, B.M. and Toivonen, H.T. (2006). A neural network model predictive controller. Journal of Process Control, 16, 937-946.

Bemporad, A. and Filippi, C. (2006). An algorithm for approximate multiparametric convex programming. Computational Optimization and Applications, 35, 87108.
Bertsekas, D.P. and Tsitsiklis, J.N. (1998). NeuroDynamic Programming. Athena Scientific, Belmont.

Chen, H. and Allgöwer, F. (1998). A quasi-infinite horizon nonlinear model predictive control scheme with guaranteed stability. Automatica, 34, 1205-1217.

Chen, S., Billings, S.A., and Grant, P.M. (1990). Nonlinear system identification using neural networks. International Journal of Control, 51, 1191-1214.

Espinosa, J., Vandewalle, J., Wertz, V. (2005). Fuzzy Logic, Identification and Predictive Control. SpringerVerlag, London.

Fiacco, A.V. (1983). Introduction to Sensitivity and Stability Analysis in Nonlinear Programming. Academic Press, Orlando, Florida.

Garcia-Nieto, S., Martinez, M., Blasco, X., and Sanchis, J. (2008). Nonlinear predictive control based on local model networks for air management in diesel engines. Control Engineering Practice, 16, 1399-1413.

Grancharova, A., Johansen, T.A., and Tøndel, P. (2007). Computational aspects of approximate explicit nonlinear model predictive control. In R. Findeisen, F. Allgöwer, and L. Biegler (eds.), Assessment and Future Directions of Nonlinear Model Predictive Control, LNCIS, volume 358, Springer-Verlag, 181-192.

Grancharova, A. and Johansen, T.A. (2009). Computation, approximation and stability of explicit feedback min-max nonlinear model predictive control. Automatica, 45, 1134-1143.

Henson, M.A. and Seborg, D.E. (1994). Adaptive nonlinear control of a $\mathrm{pH}$ neutralization process. IEEE Transactions on Control System Technology, 2, 169-183.

Johansen, T.A. (2002). On multi-parametric nonlinear programming and explicit nonlinear model predictive control. Proceedings of the IEEE Conference on Decision and Control, Las Vegas, NV, volume 3, 2768-2773.

Johansen, T.A. (2004). Approximate explicit receding horizon control of constrained nonlinear systems. $A u-$ tomatica, 40, 293-300.

Kocijan, J. and Murray-Smith, R. (2005). Nonlinear predictive control with a Gaussian process model. In R. Murray-Smith and R. Shorten (eds.), Switching and Learning in Feedback Systems, LNCS, volume 3355, Springer-Verlag, 185-200.

Ljung, L. and Glad, T. (1994). Modeling of Dynamic Systems. Prentice Hall, Englewood Cliffs, N.J.

Mayne, D.Q., Rawlings, J.B., Rao, C.V., and Scokaert, P.O.M. (2000). Constrained model predictive control: Stability and optimality. Automatica, 36, 789-814.

Michalska, H. and Mayne, D.Q. (1993). Robust receding horizon control of constrained nonlinear systems. IEEE Transactions on Automatic Control, 38, 1623-1633.

Nørgaard, M., Ravn, O., Poulsen, N.K., and Hansen, L.K. (2000). Neural Networks for Modelling and Control of Dynamic Systems. Springer, London.

Ogata, K. (1995). Discrete-time Control Systems. Englewood Cliffs, Prentice-Hall, N.J.

Parisini, T. and Zoppoli, R. (1995). A receding-horizon regulator for nonlinear systems and a neural approximation. Automatica, 31, 1443-1451.

Pistikopoulos, E.N., Georgiadis, M.C., Dua, V. (2007). Multi-parametric Programming: Theory, Algorithms, and Applications. Wiley-VCH. 